\title{
High-Throughput Multi-Frame Decoding of QC-LDPC Codes with Modified Rejection-Based Minimum Finding
}

\author{
Alireza Hasani, Lukasz Lopacinski, and Rolf Kraemer
}

\begin{abstract}
The key computation in the min-sum decoding algorithm of a Low-Density Parity-Check (LDPC) code is finding the first two minima and also the location of the first minimum among a set of messages passed from Variable Nodes (VNs) to Check Nodes $(\mathrm{CNs})$ in a Tanner graph. In this paper, we propose a modified rejection-based scheme for this task which is able to find the one-hot sequence of the minimum location instead of its index. We show that this modification effectively reduces the complexity of min-sum decoding algorithm. Additionally, we reveal a pipelining potential in such a rejection-based architecture which facilitates the multi-frame decoding of LDPC codes and therefore results in improvement in decoding throughput with bearable hardware overhead. Synthesis in an industrial $28 \mathrm{~nm}$ CMOS technology shows improved results in terms of throughput, power, and chip area.
\end{abstract}

Index Terms-LDPC code, min-sum decoding, one-hot sequence, decoding complexity, decoding throughput,

\section{INTRODUCTION}

$\mathbf{L}$ OW-Density Parity-Check (LDPC) codes are one of the selected forward-error correction candidates for nextgeneration wireless communication systems like 5G and IEEE 802.11ax. However, despite the promising performance, the decoding complexity of LDPC codes is still a barrier toward high-throughput applications of these codes. In order to address this downside of LDPC codes a great deal of effort has been expended on different aspects of them like construction, encoding, and decoding.

The major decoding method for LDPC codes is the iterative Belief Propagation (BP) algorithm run over the Tanner graph representation of these codes. BP algorithm in its primary form is conducted as a sum-product algorithm [1] in which a series of messages are successively exchanged between the nodes of the Tanner graph. Min-sum decoding algorithm [2], [3] is a simplified method compared with the sum-product scheme for BP decoding of LDPC codes replacing complex hyperbolic tangent functions with simpler minimum finding computations. More specifically, in min-sum decoding algorithm the computation of messages passed from CNs to VNs in a Tanner graph during decoding is targeted for simplification, and a $\mathrm{CN}$ finds the minimum value among the messages receiving from its

The authors are with IHP - Leibniz-Institut für innovative Mikroelektronik, Frankfurt (Oder), Germany (e-mail: \{hasani, lopacinski, kraemer\}@ihpmicroelectrinics.com)

A. Hasani and R. Kraemer are also with the Department of Electrical and Computer Engineering, Brandenburg university of technology, CottbusSenftenberg, Cottbus, Germany. neighbor VNs instead of computing complicated hyperbolic tangent functions.

After a more detailed introduction of min-sum decoding in section II it becomes evident that the essential computation of this decoding scheme is finding the first and second minima together with the index (or location) of the first minimum among some binary values. This computation, although simpler than the original computations in sum-product algorithm, should still be performed with the most simplest approach in order to avoid overall decoding complexity. To this goal, different approaches have been proposed specifically for application to min-sum decoding which [4] gives an overview of them.

In this regard, authors in [5] proposed a hardware-friendly rejection-based technique which is able to accomplish this task faster than the former techniques, only with insubstantial increase in circuit area. Additionally, we proposed in [4] a modification to this method which suggests to use onehot sequence representation for the location of minimum instead of its binary index, claiming that this modification has implementation benefits. More specifically, the rejectionbased scheme of [5] finds the index of the first minimum, while in our modified approach the one-hot sequence of the first minimum is found. In this paper, we first re-investigate this modification and show how the rejection-based technique can be modified to output one-hot sequence of the minimum. Besides, an important pipelining capacity of this method is revealed which is able to boost decoding throughput while maintaining the overall hardware overhead acceptable and not increasing the consumed energy per bit. The proposed idea of multi-frame decoding with the modified rejectionbased scheme is synthesized in an industrial $28 \mathrm{~nm}$ CMOS technology. Results from this synthesis reveal a considerable improvement in throughput, power consumption, and also chip area of the min-sum decoder.

The organization of the paper is as follows. Section II provides the necessary preliminaries, specifically about minsum decoding algorithm and also rejection-based method for finding the first two minima. In section III, the modified rejection-based scheme is introduced and its pipelining capacity for boosting decoding throughput is revealed in section IV. Section $V$ is devoted to simulation and synthesis results, and final conclusions are made in section VI. 


\section{PRELIMINARIES}

\section{A. Quasi-Cyclic LDPC (QC-LDPC) Codes and Layered De- coding $(L D)$ Schedule}

In a nutshell, the codewords of a QC-LDPC code are spanned by a sparse Row-Column (RC)-constrained [6] matrix composed of only Circulant-Permutation Matrices (CPMs) and zero matrices. A CPM can be assumed as an identity matrix in that all the rows have been shifted cyclically. Fig. 1-(a) shows an example Parity-Check Matrix (PCM) of a QC-LDPC code.

$\mathrm{BP}$ is the major decoding algorithm of LDPC codes, performed based on the Tanner graph representation of them. The Tanner graph of an example LDPC code has been shown in Fig. 2 which is composed of two sets of nodes and a number of connections between them. The gray circles, called VNs, represent the code bits or columns of the PCM, while the white circles, called CNs, denote the check sums or rows of the PCM. During the BP algorithm, reliability messages are successively passed between VNs and CNs in an attempt to obtain probabilities expressing whether a given symbol in a received codeword is ' 1 ' or ' 0 '. The resulting sequence will then have the maximum probability according to the received soft-decision sequence. We assume $Z_{j, l}$ as a Variable-to-Check (VTC) message passed from $l^{t h} \mathrm{VN}$ to $j^{t h} \mathrm{CN}$ and $L_{j, l}$ as a Check-to-Variable (CTV) message passed from $j^{\text {th }} \mathrm{CN}$ to $l^{\text {th }}$ $\mathrm{VN}$.

The rules governing the order of the exchange of messages between the nodes of a Tanner graph is referred to as the schedule of the BP algorithm. One basic possibility is the flood-like schedule, in which, in each iteration, firstly all the VNs update their messages and send them to $\mathrm{CNs}$ and then CNs update their own messages and send them back to VNs. Flood schedule facilitates a fully parallel decoding architecture, yet at the cost of high interconnect complexity. LD is rather a partially parallel schedule which can propose faster convergence rate, improved coding gain, and reduced hardware overhead over flood schedule with lower decoding complexity [7]. In LD schedule, the rows in the PCM are split in layers, and then the $\mathrm{BP}$ algorithm runs over layers in successive order. In other words, each iteration of the algorithm is split into several sub-iterations, during each one reliability messages are exchanged between CNs of that layer and their neighbor VNs. At the end of each sub-iteration the updated reliability messages are handed down to the next layer. Accordingly, only a subset of CNs and VNs participate in each sub-iteration, and layers are processed successively from top to down the PCM.

The complexity of LD schedule is lowered if the layers in the PCM have only single- or zero-weight columns. QC-LDPC codes have inherently such a property, if each row of CPMs is considered as a layer. Moreover, by the shuffling idea proposed in [8] the implementation complexity of LD is further simplified, since it suffices to define only the connections of the first layer of the shuffled PCM for decoding. The shuffling only interleaves the row orders and thus does not degrade the BitError Rate (BER) performance. More details on the principles of shuffling, how it is performed and how it is beneficial are found in [8]. Fig. 1-(b) shows the shuffled version of the example PCM.

\section{B. Min-Sum Decoding}

Min-sum decoding is a simplified variant of BP algorithm, in which the magnitude of a CTV message $\left|L_{j, l}\right|$ is approximated by the minimum of the magnitude of all the VTC messages $\left|Z_{j, l^{\prime}}\right|$ arriving in $s_{j}$ from all its neighbors except $v_{l}$. For example, in Fig. 2, CTV messages sent from $s_{1}$ to its neighbor $\mathrm{VNs} v_{0}, v_{1}$ and $v_{3}$ have the magnitude of $\left|L_{1,0}\right|=\min \left(\left|Z_{1,1}\right|,\left|Z_{1,3}\right|\right),\left|L_{1,1}\right|=\min \left(\left|Z_{1,0}\right|,\left|Z_{1,3}\right|\right)$ and $\left|L_{1,3}\right|=\min \left(\left|Z_{1,0}\right|,\left|Z_{1,1}\right|\right)$. This implies that the CTV messages sent from a $\mathrm{CN}$ to its neighbor VNs are either the first or second minimum among the VTC messages that CN has just received. For instance, if one assumes that the relation $\left|Z_{1,0}\right|<\left|Z_{1,1}\right|<\left|Z_{1,3}\right|$ holds for the previous example, then it results in $\left|L_{1,0}\right|=\left|Z_{1,1}\right|,\left|L_{1,1}\right|=\left|Z_{1,0}\right|$ and $\left|L_{1,3}\right|=\left|Z_{1,0}\right|$. Accordingly, the task of a $\mathrm{CN}$ processing unit in a min-sum decoding algorithm reduces to finding the first and second minimum among the incoming VTC messages along with the location of the first minimum, with the latter needed, because the CTV message at that location is simply valuated as the second minimum and all the other CTV messages as the first minimum.

\section{Rejection-Based First Two Minima Finding}

Several works have been thus far devoted to the efficient methods for finding the first two minima and the index of the minimum exclusively for min-sum LDPC decoders [5], [9]-[18]. Among them, tree-based architectures have been of greater importance, whose latest variant is the rejection-based method proposed by the authors in [5]. Let $x_{0}, \ldots, x_{\rho-1}$ be the $\rho$ binary fixed-point numbers, each $K$ bits long, whose first two minima $\min _{1}$ and $\min _{2}$ are to be found. In this method, two $3 \times 2$-MIN and $4 \times 2$-MIN modules, shown in Fig. 3 serve as the fundamental building blocks for constructing larger modules with arbitrary number of inputs. In these modules in the first step all the two combinations of the $\rho=3$ or 4 numbers are compared in pair by the MIN units, thus yielding the flags $a, b, c$ in $3 \times 2$-MIN or $a, b, c, d, e, f$ in $4 \times 2$-MIN modules. These flags are then used to form the select bits of the multiplexers, according to the relations stated at the bottom of the two modules. In these relations, the signs ".", "+" and "bar" represent logical operations of AND, OR and complement respectively. Note further that each MIN unit in these modules outputs " 1 " if its upper input is bigger than its lower input and "0" otherwise.

Building larger modules is viable by proper configuration of the two fundamental $3 \times 2$-MIN and $4 \times 2$-MIN modules. Fig. 4 shows such configurations for the five-, six-, seven- and eight-input modules. It should be noted that the outputs $O_{1}$ and $O_{2}$ in figures 3 and 4 are not sorted, meaning that it may be $\min _{1}=O_{1}$ and $\min _{2}=O_{2}$ or vice versa. However, the index output specifies the location of $\min _{1}$. The index output in this method is in the form of binary representation of the location of $\min _{1}$. For example, if $\rho=4$, the index output will be either " $00 "$, " $01 "$, " $10 "$ or " 11 " in order to specify the index of the minimum. 
$\boldsymbol{H}_{q c}=\left[\begin{array}{cccccccc|cccccccc}0 & 0 & 0 & \mid & 0 & 0 & 1 & \mid & 0 & 1 & 0 & \mid & 1 & 0 & 0 \\ 0 & 0 & 0 & \mid & 1 & 0 & 0 & \mid & 0 & 0 & 1 & \mid & 0 & 1 & 0 \\ 0 & 0 & 0 & \mid & 0 & 1 & 0 & \mid & 1 & 0 & 0 & \mid & 0 & 0 & 1 \\ - & - & - & - & - & - & - & - & - & - & - & - & - & - & - \\ 0 & 0 & 1 & \mid & 0 & 0 & 0 & \mid & 1 & 0 & 0 & \mid & 0 & 1 & 0 \\ 1 & 0 & 0 & \mid & 0 & 0 & 0 & \mid & 0 & 1 & 0 & \mid & 0 & 0 & 1 \\ 0 & 1 & 0 & \mid & 0 & 0 & 0 & \mid & 0 & 0 & 1 & \mid & 1 & 0 & 0 \\ - & - & - & - & - & - & - & - & -- & - & - & - & - & - & - \\ 0 & 1 & 0 & \mid & 1 & 0 & 0 & \mid & 0 & 0 & 0 & \mid & 0 & 0 & 1 \\ 0 & 0 & 1 & \mid & 0 & 1 & 0 & \mid & 0 & 0 & 0 & \mid & 1 & 0 & 0 \\ 1 & 0 & 0 & \mid & 0 & 0 & 1 & \mid & 0 & 0 & 0 & \mid & 0 & 1 & 0 \\ - & - & - & - & - & - & - & - & - & - & - & - & - & - & - \\ 1 & 0 & 0 & \mid & 0 & 1 & 0 & \mid & 0 & 0 & 1 & \mid & 0 & 0 & 0 \\ 0 & 1 & 0 & \mid & 0 & 0 & 1 & \mid & 1 & 0 & 0 & \mid & 0 & 0 & 0 \\ 0 & 0 & 1 & \mid & 1 & 0 & 0 & \mid & 0 & 1 & 0 & \mid & 0 & 0 & 0\end{array}\right] \rightarrow \boldsymbol{H}_{q c}^{s h}=\left[\boldsymbol{H}_{q c}^{s h,(2)}\left[\begin{array}{l}\boldsymbol{H}_{q c}^{s h,(1)} \\ -1\end{array}\right]=\left[\begin{array}{cccccccccccccccc}0 & 0 & 0 & \mid & 0 & 0 & 1 & \mid & 0 & 1 & 0 & \mid & 1 & 0 & 0 \\ 0 & 0 & 1 & \mid & 0 & 0 & 0 & \mid & 1 & 0 & 0 & \mid & 0 & 1 & 0 \\ 0 & 1 & 0 & \mid & 1 & 0 & 0 & \mid & 0 & 0 & 0 & \mid & 0 & 0 & 1 \\ 1 & 0 & 0 & \mid & 0 & 1 & 0 & \mid & 0 & 0 & 1 & \mid & 0 & 0 & 0 \\ - & - & - & - & - & - & - & - & - & - & - & - & - & - & - \\ 0 & 0 & 0 & \mid & 1 & 0 & 0 & \mid & 0 & 0 & 1 & \mid & 0 & 1 & 0 \\ 1 & 0 & 0 & \mid & 0 & 0 & 0 & \mid & 0 & 1 & 0 & \mid & 0 & 0 & 1 \\ 0 & 0 & 1 & \mid & 0 & 1 & 0 & \mid & 0 & 0 & 0 & \mid & 1 & 0 & 0 \\ 0 & 1 & 0 & \mid & 0 & 0 & 1 & \mid & 1 & 0 & 0 & \mid & 0 & 0 & 0 \\ - & - & - & - & - & - & - & - & - & - & - & - & - & - & - \\ 0 & 0 & 0 & \mid & 0 & 1 & 0 & \mid & 1 & 0 & 0 & \mid & 0 & 0 & 1 \\ 0 & 1 & 0 & \mid & 0 & 0 & 0 & \mid & 0 & 0 & 1 & \mid & 1 & 0 & 0 \\ 1 & 0 & 0 & \mid & 0 & 0 & 1 & \mid & 0 & 0 & 0 & \mid & 0 & 1 & 0 \\ 0 & 0 & 1 & \mid & 1 & 0 & 0 & \mid & 0 & 1 & 0 & \mid & 0 & 0 & 0\end{array}\right]\right.$

(a)

(b)

Fig. 1. (a) PCM of a (12,4)-QC-LDPC code; (b) its shuffled version.

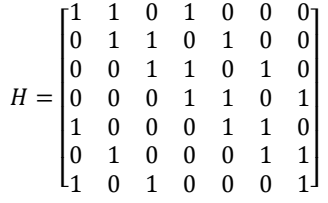

(a)

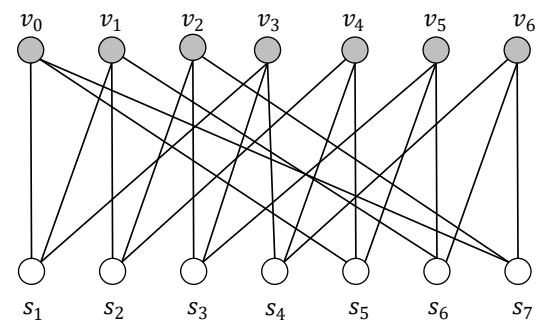

(b)

Fig. 2. (a) The PCM of a (7,3)-LDPC code; (b) Its Tanner graph.

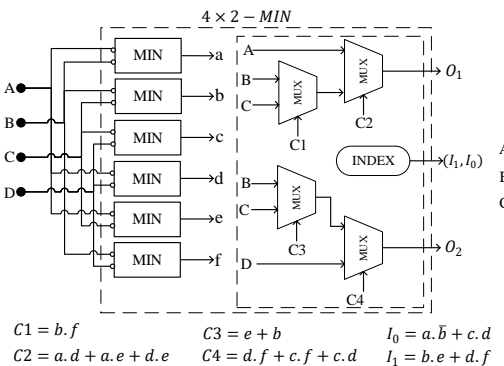

(a)

Fig. 3. Fundamental sorting modules in rejection-based technique (a) Fourinput two-output module; (b) Three-input two-output module [5].

\section{Modified Rejection-BASED Scheme}

Besides the binary representation, there is an alternative way of specifying the location of $\min _{1}$, referred to as the one-hot sequence. A one-hot sequence is a $\rho$-bit binary sequence, in that all the bits except one are ' 0 '. The only ' 1 ' in the sequence resides in the same location as the index of the $\min _{1}$. For example, for $\rho=4$, the one-hot sequence may be "1000", "0100", "0010" or "0001", in each case the location of '1' specifies the index of the $\min _{1}$.
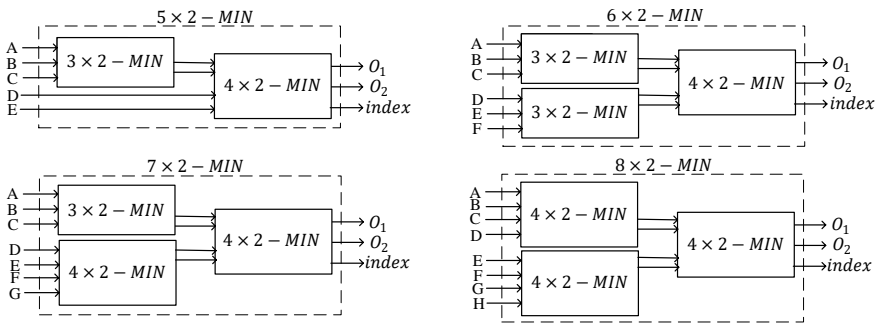

Fig. 4. Configuration of fundamental modules to build larger comparator modules based on rejection scheme for $\rho=5,6,7$ and 8 .

We claim that the one-hot sequence is the superior way of locating $\min _{1}$ instead of its binary representation, since it simplifies the overall min-sum decoding algorithm. To argue that, the schematic diagram of the relevant part of the minsum decoding scheme is sketched in Fig. 5. This diagram illustrates how CTV messages are computed from the received VTC messages at a $\mathrm{CN}$ with degree 4. Among the four received VTC messages, $\min _{1}, \min _{2}$ and location of $\min _{1}$ must be determined. Then, the value of the CTV message at the location of first minimum takes the value of $\min _{2}$ and all the other CTV messages take the value of $\min _{1}$. In Fig. 5-a the index output is the location of $\min _{1}$ specified as the one-hot sequence. As shown, as many multiplexers as the weight of the processing $\mathrm{CN}$, in this example 4, are needed, and the bits of index output, namely inde $x_{i}$ are used directly as the select bits of the multiplexers. If a select bit is '0', $S_{1}$ which is $\min _{1}$ is selected, and otherwise $S_{2}$ which is $\min _{2}$. Hence, only one of the multiplexers outputs $\min _{2}$ and all the others output $\min _{1}$. In contrast, in Fig. 5-b the binary representation of $\min _{1}$ location is provided. As a result, a set of additional comparators which have been boldfaced in the figure are required to produce the select bits of the multiplexers. A comparator outputs '1' only if its two inputs equal. The first input of the comparators are respectively the different possibilities of the index, in this example "00", "01", "10", and "11". Accordingly, only one of the comparators will have a ' 1 ' in output, leading to $\min _{2}$ to appear at the output of the multiplexer connected to that comparator. The other comparators will have ' 0 ' in output, leading to $\min _{1}$ to appear 

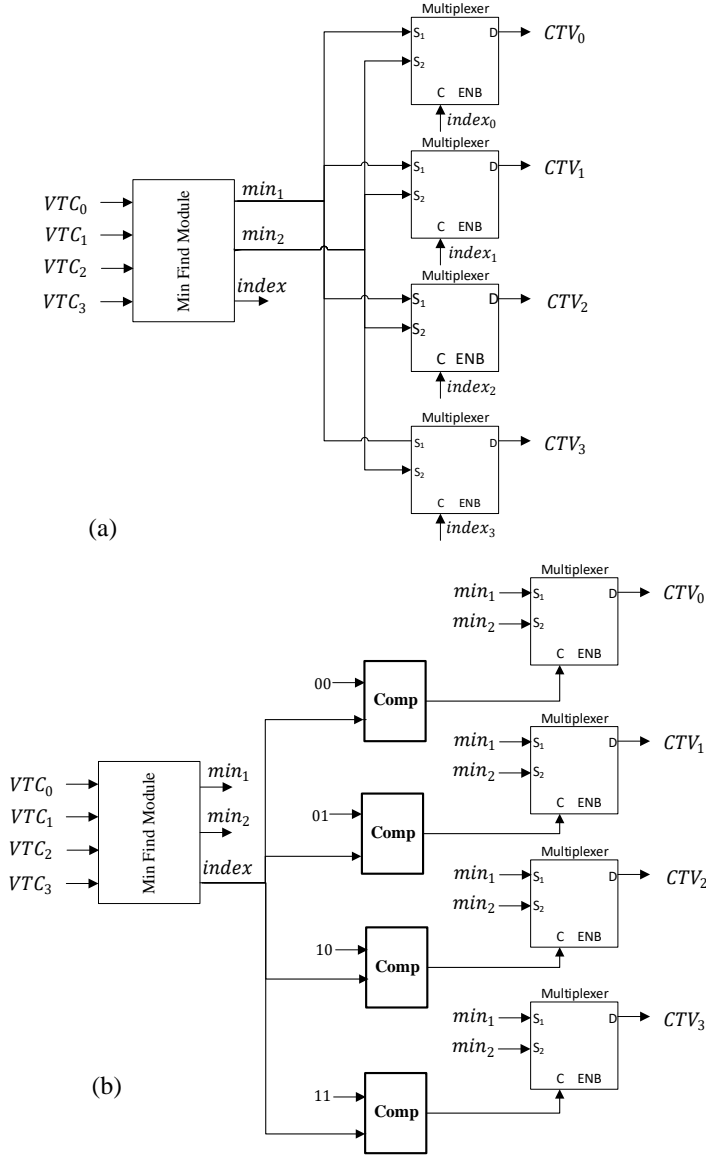

Fig. 5. Computation of CTV messages in a min-sum decoding algorithm, when the index of $\min _{1}$ is specified by (a) one-hot sequence, and (b) binary representation.

at the output of their multiplexers. Based on this argument, one can claim that one-hot sequence is the preferred way for representation of the location of $\min _{1}$ in min-sum decoding method, since the need for extra comparators is eliminated.

The two $3 \times 2$-MIN and $4 \times 2$-MIN circuits in Fig. 3 can be modified to output the one-hot sequence of the location of $\min _{1}$ instead of its binary index. Fig. 6 illustrates the modified circuits in them the outputs $I 0, I 1, I 2$, and $I 3$ are the bits of the one-hot sequence. These bits are computed based on the flag bits output by MIN units. The corresponding relations for deriving these bits are stated at the bottom of this figure, and they are developed based on the truth tables I and II, respectively for $3 \times 2$-MIN and $4 \times 2$-MIN modified circuits. These relations have been expressed in an optimized form which is straightforward to do so with using basic methods for optimized implementation of logic functions like Karnaugh map [19] or one of other advanced methods.

In our modified rejection-based scheme only the fundamental $3 \times 2$-MIN and $4 \times 2$-MIN circuits are modified to output the location of $\min _{1}$ as a one-hot sequence. However, building larger modules with arbitrary number of inputs follows the same procedure as exemplified for the original scheme in Fig. 4.

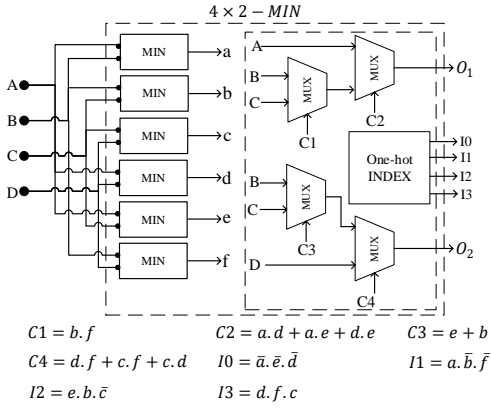

(a)

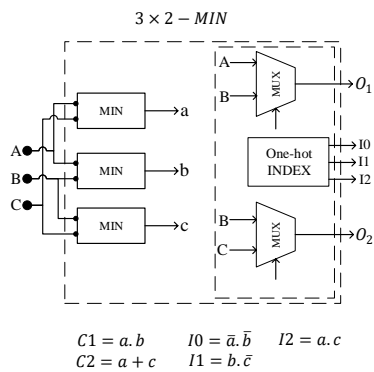

(b)
Fig. 6. Fundamental sorting units in modified rejection-based technique (a) Four-input two-output module; (b) Three-input two-output module.

TABLE I

TRUTH TABLE FOR THE $3 \times 2$-MIN MODULE OF THE MODIFIED REJECTION-BASED METHOD.

\begin{tabular}{c|ccc|cc|ccc}
\hline Status & $a$ & $b$ & $c$ & $C 1$ & $C 2$ & $I 0$ & $I 1$ & $I 2$ \\
\hline$A<B<C$ & 0 & 0 & 0 & 0 & 0 & 1 & 0 & 0 \\
\hline$A<C<B$ & 0 & 0 & 1 & 0 & 1 & 1 & 0 & 0 \\
\hline$B<A<C$ & 0 & 1 & 0 & 0 & 0 & 0 & 1 & 0 \\
\hline$B<C<A$ & 1 & 1 & 0 & 1 & 1 & 0 & 1 & 0 \\
\hline$C<A<B$ & 1 & 0 & 1 & 0 & 1 & 0 & 0 & 1 \\
\hline$C<B<A$ & 1 & 1 & 1 & 1 & 1 & 0 & 0 & 1 \\
\hline
\end{tabular}

TABLE II

TRUTH TABLE FOR THE $4 \times 2$-MIN MODULE OF THE MODIFIED REJECTION-BASED METHOD.

\begin{tabular}{c|cccccc|cccc|cccc}
\hline Status & $a$ & $b$ & $c$ & $d$ & $e$ & $f$ & $C 1$ & $C 2$ & $C 3$ & $C 4$ & $I 0$ & $I 1$ & $I 2$ & $I 3$ \\
\hline$A<B<C<D$ & 0 & 0 & 0 & 0 & 0 & 0 & 0 & 0 & 0 & 0 & 1 & 0 & 0 & 0 \\
\hline$A<B<D<C$ & 0 & 0 & 1 & 0 & 0 & 0 & 0 & 0 & 0 & 0 & 1 & 0 & 0 & 0 \\
\hline$A<C<B<D$ & 0 & 1 & 0 & 0 & 0 & 0 & 0 & 0 & 1 & 0 & 1 & 0 & 0 & 0 \\
\hline$A<C<D<B$ & 0 & 1 & 0 & 0 & 0 & 1 & 1 & 0 & 1 & 0 & 1 & 0 & 0 & 0 \\
\hline$A<D<B<C$ & 0 & 0 & 1 & 0 & 0 & 1 & 0 & 0 & 0 & 1 & 1 & 0 & 0 & 0 \\
\hline$A<D<C<B$ & 0 & 1 & 1 & 0 & 0 & 1 & 1 & 0 & 1 & 1 & 1 & 0 & 0 & 0 \\
\hline$B<A<C<D$ & 1 & 0 & 0 & 0 & 0 & 0 & 0 & 0 & 0 & 0 & 0 & 1 & 0 & 0 \\
\hline$B<A<D<C$ & 1 & 0 & 1 & 0 & 0 & 0 & 0 & 0 & 0 & 0 & 0 & 1 & 0 & 0 \\
\hline$B<C<A<D$ & 1 & 0 & 0 & 0 & 1 & 0 & 0 & 1 & 1 & 0 & 0 & 1 & 0 & 0 \\
\hline$B<C<D<A$ & 1 & 0 & 0 & 1 & 1 & 0 & 0 & 1 & 1 & 0 & 0 & 1 & 0 & 0 \\
\hline$B<D<A<C$ & 1 & 0 & 1 & 1 & 0 & 0 & 0 & 1 & 0 & 1 & 0 & 1 & 0 & 0 \\
\hline$B<D<C<A$ & 1 & 0 & 1 & 1 & 1 & 0 & 0 & 1 & 1 & 1 & 0 & 1 & 0 & 0 \\
\hline$C<A<B<D$ & 0 & 1 & 0 & 0 & 1 & 0 & 0 & 0 & 1 & 0 & 0 & 0 & 1 & 0 \\
\hline$C<A<D<B$ & 0 & 1 & 0 & 0 & 1 & 1 & 1 & 0 & 1 & 0 & 0 & 0 & 1 & 0 \\
\hline$C<B<A<D$ & 1 & 1 & 0 & 0 & 1 & 0 & 0 & 1 & 1 & 0 & 0 & 0 & 1 & 0 \\
\hline$C<B<D<A$ & 1 & 1 & 0 & 1 & 1 & 0 & 0 & 1 & 1 & 0 & 0 & 0 & 1 & 0 \\
\hline$C<D<A<B$ & 0 & 1 & 0 & 1 & 1 & 1 & 1 & 1 & 1 & 1 & 0 & 0 & 1 & 0 \\
\hline$C<D<B<A$ & 1 & 1 & 0 & 1 & 1 & 1 & 1 & 1 & 1 & 1 & 0 & 0 & 1 & 0 \\
\hline$D<A<B<C$ & 0 & 0 & 1 & 1 & 0 & 1 & 0 & 0 & 0 & 1 & 0 & 0 & 0 & 1 \\
\hline$D<A<C<B$ & 0 & 1 & 1 & 1 & 0 & 1 & 1 & 0 & 1 & 1 & 0 & 0 & 0 & 1 \\
\hline$D<B<A<C$ & 1 & 0 & 1 & 1 & 0 & 1 & 0 & 1 & 0 & 1 & 0 & 0 & 0 & 1 \\
\hline$D<B<C<A$ & 1 & 0 & 1 & 1 & 1 & 1 & 0 & 1 & 1 & 1 & 0 & 0 & 0 & 1 \\
\hline$D<C<A<B$ & 0 & 1 & 1 & 1 & 1 & 1 & 1 & 1 & 1 & 1 & 0 & 0 & 0 & 1 \\
\hline$D<C<B<A$ & 1 & 1 & 1 & 1 & 1 & 1 & 1 & 1 & 1 & 1 & 0 & 0 & 0 & 1 \\
\hline & & & & & & & & & & & \\
\hline
\end{tabular}




\section{Multi-Frame Decoder Architecture}

In this section, we reveal a potential for improving decoding throughput of LDPC codes. The flowchart of the decoding procedure is shown in Figure 7. Decoding begins with fetching a new sequence whose corresponding codeword is sought. This sequence is initially checked if it satisfies the paritycheck equations. If yes, it means that it is already an error-free codeword and a new sequence can be taken in for decoding. Otherwise, it enters the decoding loop until it is decoded successfully or the preset number of iterations is reached and decoding fails. Each round of the decoding loop is in fact one sub-iteration performed on a layer of a PCM.

Each processing step in the decoding loop can be implemented in one clock cycle, except for the step of updating CTV messages, which is equivalent to finding the first two minima and also the location of the first minimum among a multiple of VTC messages. The rejection-based approach outlined in section II-C or its modified version proposed in section III do not carry out their task in one step. Therefore, they need more than one clock cycle to perform their task if reserving one clock cycle for each step.

By examining the structure of $3 \times 2$-MIN and $4 \times 2$-MIN modules in Fig. 3 or 6 it is deduced that they need two clock cycles for their operation. In the first clock cycle the pairwise comparisons of the inputs $\mathrm{A}, \mathrm{B}, \mathrm{C}$ and $\mathrm{D}$ are carried out and the flags $a, b, c, d, e$ and $f$ are formed. Then, in the second clock cycle the outputs of the module are generated. When building modular circuits with 5-8 inputs, as exemplified by Fig. 4, two levels of fundamental units are needed. This results in the latency of $2 \times 2$ clock cycles. Likewise, when building larger modular circuits with 9-16 inputs, the number of levels of fundamental modules will be 3 and hence they need $3 \times 2$ clock cycles. By deduction, we can state that the number of levels of fundamental modules needed for building a configuration with $\rho$ inputs is $\left\lceil\log _{2} \rho\right\rceil-1$. This yields the latency of $2\left(\left\lceil\log _{2} \rho\right\rceil-1\right)+1$. The one extra clock cycle is required to perform the final sorting between the two outputs of the module in order to determine which one is $\min _{1}$ and which one is $\min _{2}$.

The multi-level structure of the (modified) rejection-based scheme can be used smartly to increase decoding throughput without considerable hardware overhead. In this multi-level structure when one layer is given input, its previous layers are already done and they are in idle mode. Consider e.g. the configuration in Fig. 8, i.e., a $16 \times 2$-MIN module requiring 7 clock cycles to finish its work. During the first two clock cycles the four $4 \times 2$-MIN modules at level- 1 are active. During the clock cycles 3 and 4 these two modules stand by and the two $4 \times 2$-MIN modules at level-2 start operating. The last three clock cycles belong then to the single $4 \times 2$-MIN module at level-3. Apparently, for this architecture, the flow of inputs could become non-stop and the module can take new inputs every clock cycles.

The Register-Transfer Level (RTL) simulation of the 16input modified rejection-based module of Fig. 8 is illustrated in Fig. 9. The figure shows that the module needs initially 7 clock cycles to output the result of the first inputs. But afterward, it is given input every clock cycles, and it gives output every clock cycles as well. In this figure, "clk" is the clock signal and the 16 inputs are denoted by "d1" to "d16". The signals "O1" and "O2" are $\min _{1}$ and $\min _{2}$ respectively and "min_index" is the location of $\min _{1}$ as a one-hot sequence.

This multi-frame processing although allows for decoding several sequences at the same time, it comes with the downside of additional overhead. When several sequences are taken in for decoding, it is not known which one will be converged to a valid codeword and leave the decoder sooner than the others. Therefore, the decoded sequences will be most likely out of the order with which they have entered the decoder and a need for numbering the sequences and tracking their order is inevitable. On one side, this introduces additional hardware overhead to accomplish the numbering and tracking of the sequences. On the other side, it imposes a restriction on the maximum number of sequences which can be decoded at the same time. The latter is due to the memory size which is needed to order the decoded codewords. We refer to this parameter as the multiplicity factor which is a positive integer and determines the maximum number of sequences that can be decoded simultaneously. When multiplicity factor is one, there is not any cohesiveness in the decoder and a sequence enter decoder only if the previous sequence has left it. For multiplicity factor bigger than one the decoding throughput is expected to increase however with the price of hardware overhead.

\section{Performance Analysis and Comparison Results}

We have synthesized the multi-frame decoding algorithm for decoding four IEEE 802.11 QC-LDPC codes in an industrial $28 \mathrm{~nm}$ technology. The synthesis has been conducted with multiplicity factor of 1,4 , and 8 , for each code. This parameter indicates the maximum number of frames that can be decoded simultaneously. Corresponding results are found in table III.

All syntheses have been conducted with $(6,2)$ quantization bits, meaning that floating-point values are converted to 6bit fixed-point values in which 2 bits are dedicated to the fractional part and the remaining 4 bits to integer part. This specific choice is based on MATLAB simulation results shown in Fig. 10 that shows the BER performance degradation with $(6,2)$-bit fixed-point values compared with the case of floatingpoint values is negligible. The $E_{b} / N_{0}$ values in Table III for which the BER is 1e-7 are also derived based on the simulations in Fig. 10. The average number of iterations needed by the decoder to reach a specific BER performance is also important to consider when examining the effect of quantization. The average number of iterations related to the BER curves of Fig. 10 are shown in Fig. 11 also showing that the increase in number of iterations is also negligible when using quantized messages during decoding.

As the multiplicity factor rises from 1 to 4 and then 8 , throughput also increases largely, while the energy efficiency remains nearly unaltered. Besides, the increase in chip area is with a smaller rate than the increase rate of throughput. To show this evidently Fig. 12 depicts the bar graph of the increase rate of both throughput and chip area for the four 


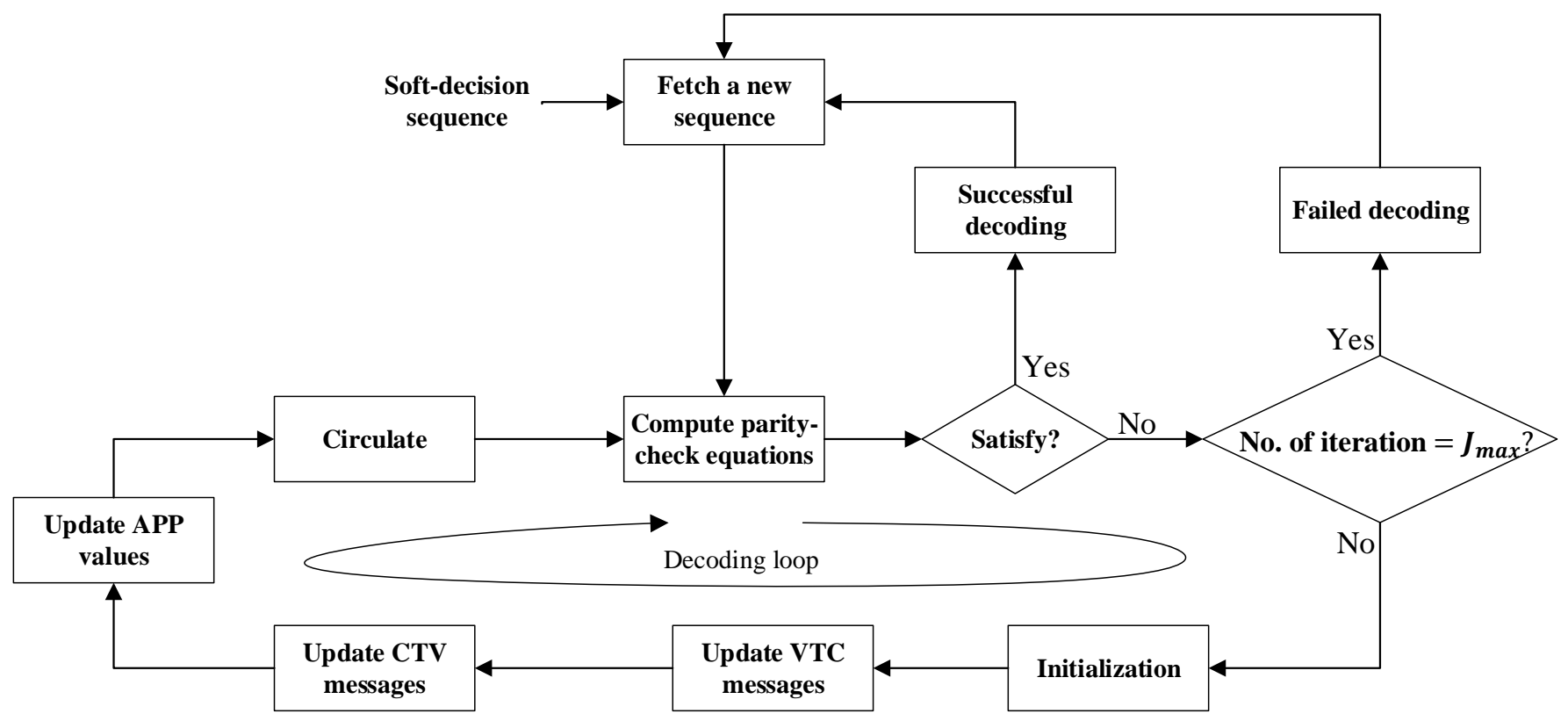

Fig. 7. Flowchart of the LD algorithm [20].

TABLE III

IMPLEMENTATION RESULTS FOR THE PROPOSED HIGH-THROUGHPUT DECODER WITH MULTI-FRAME PROCESSING CAPABILITY IN 28 NM TECHNOLOGY WITH $J_{\max }=5$, AND $(6,2)$ QUANTIZATION.

\begin{tabular}{|c|c|c|c|c|c|c|}
\hline Code & $\begin{array}{l}\text { Eb/N0 [dB] } \\
@ B E R=1 e-7\end{array}$ & $\begin{array}{l}\text { Multiplicity } \\
\text { Factor }\end{array}$ & Area $\left[\mathrm{mm}^{2}\right]$ & $\begin{array}{l}f_{c l k} \\
{[G H z]}\end{array}$ & $\begin{array}{c}\text { Throughput } \\
{[G b / s]}\end{array}$ & $\begin{array}{c}\text { Energy efficiency } \\
{[p J / b]}\end{array}$ \\
\hline \multirow{3}{*}{$\begin{array}{l}\text { IEEE 802.11 } \\
(648,324)\end{array}$} & \multirow{3}{*}{2.95} & 1 & 0.038 & 3.33 & 3.68 & 35.54 \\
\hline & & 4 & 0.089 & 2.5 & 9.5 & 36.04 \\
\hline & & 8 & 0.149 & 2.5 & 17.13 & 35.7 \\
\hline \multirow{3}{*}{$\begin{array}{c}\text { IEEE 802.11 } \\
(648,432)\end{array}$} & \multirow{3}{*}{3.84} & 1 & 0.041 & 3.33 & 4.52 & 31.01 \\
\hline & & 4 & 0.092 & 3.33 & 15.88 & 31.02 \\
\hline & & 8 & 0.155 & 3.33 & 28.4 & 36.5 \\
\hline \multirow{3}{*}{$\begin{array}{l}\text { IEEE 802.11 } \\
(648,486)\end{array}$} & \multirow{3}{*}{4.86} & 1 & 0.040 & 3.33 & 6.49 & 19.61 \\
\hline & & 4 & 0.092 & 3.33 & 21.71 & 19.49 \\
\hline & & 8 & 0.158 & 3.33 & 39.17 & 18.34 \\
\hline \multirow{3}{*}{$\begin{array}{l}\text { IEEE 802.11 } \\
(648,540)\end{array}$} & \multirow{3}{*}{5.5} & 1 & 0.043 & 3.33 & 7.87 & 18.49 \\
\hline & & 4 & 0.094 & 3.33 & 24.74 & 18.52 \\
\hline & & 8 & 0.160 & 3.33 & 43.29 & 15.65 \\
\hline
\end{tabular}

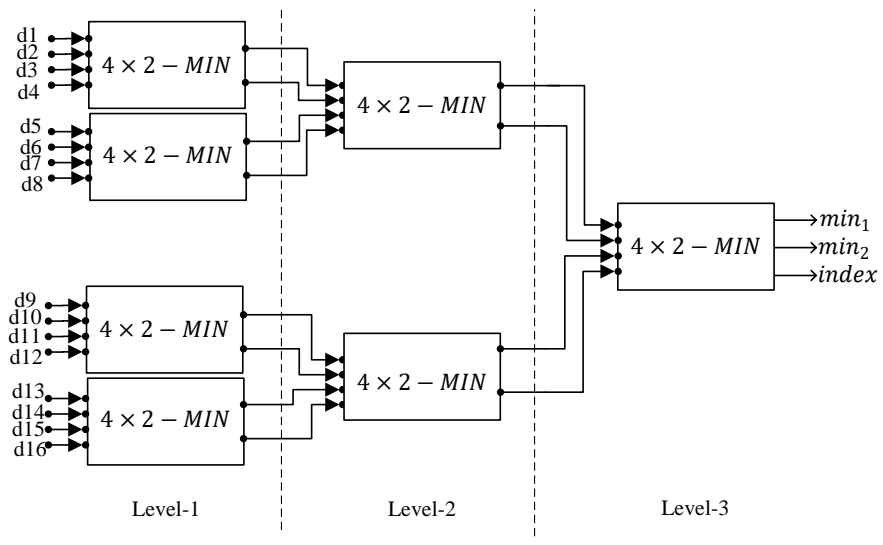

Fig. 8. (Modified) Rejection-based $16 \times 2$-MIN module.

examples. This approves that the idea of multi-frame decoding effectively improves the overall decoding throughput with a bearable hardware overhead.

Comparing our results with the previous works on high- throughput LDPC decoders is performed in Table IV. In order to have a fair comparison the achieved throughput in each case has been normalized with respect to chip area. As a result of multi-frame decoding idea the normalized throughput has been improved considerably in our work, and consumed power has decreased substantially.

\section{CONCLUSION}

A modified rejection-based scheme for finding the first two minima and location of the first minimum in a minsum decoding algorithm of an LDPC code was proposed. In this modified method the location of the minimum is derived as a one-hot sequence instead of an index, thus leading to simplification of the min-sum decoding. In addition, rejectionbased scheme allows for further pipelining of the decoding procedure and thus a multi-frame decoding architecture. This idea can effectively increase decoding throughput without prohibitive hardware overhead and thus is a practical idea. Our synthesis results in an industrial 28nm CMOS technology 


\begin{tabular}{|c|c|c|c|c|c|c|c|c|c|c|}
\hline \multirow{2}{*}{\multicolumn{11}{|c|}{$\mathrm{clk}$}} \\
\hline & & & & & & & & & & \\
\hline $\mathrm{d} 1$ & 10 'h-2 & 10 h005 & $10 \mathrm{~h} 022$ & 10 'h3E9 & 10 ' & 10 h3F6 & 10 h027 & 10 h3D9 & 10 h016 & $10 \mathrm{~h} 014$ \\
\hline $\mathrm{d} 2$ & 10 h047 & $10 \mathrm{~h} 3 \mathrm{D} 8$ & $10 \mathrm{ho1A}$ & $10 \mathrm{~h} 02 \mathrm{~B}$ & 10 h016 & 10 h033 & $10 \mathrm{~h} 015$ & 10 h3E9 & 10'h3E3 & 10 hoof \\
\hline d3 & $10 \mathrm{~h} 018$ & $10 \mathrm{~h} 3 \mathrm{DD}$ & 10'h03B & 10 h3F6 & $10 \mathrm{~h} 01 \mathrm{~B}$ & $10 \mathrm{~h} 024$ & $10 \mathrm{~h} 3 \mathrm{FD}$ & 10 'h3F4 & 10 h030 & $10 \mathrm{~h} 3 \mathrm{D} 7$ \\
\hline d4 4 & $10 \mathrm{~h} 3 \mathrm{DE}$ & $10 \mathrm{~h} 3 \mathrm{D} 3$ & $10 \mathrm{~h} 3 \mathrm{~F} 3$ & $10 \mathrm{~h} 3 \mathrm{D} 8$ & 10'h3E3 & $10 \mathrm{~h} 3 \mathrm{CA}$ & $10 \mathrm{ho10}$ & $10 \mathrm{~h} 01 \mathrm{~A}$ & $10 \mathrm{ho15}$ & $10 \mathrm{ho08}$ \\
\hline d5 & $10^{\prime}$ h039 & 10'h3D9 & $10 \mathrm{~h} 018$ & 10 h3CC & $10 \mathrm{~h} 3 \mathrm{CF}^{\dagger}$ & $10 \mathrm{~h} 3 \mathrm{DE}$ & 10'h3E4 & 10 h3E9 & 10 'h023 & 10 ' 3 E9 \\
\hline$d 6$ & $10 \mathrm{~h} 025$ & $10 \mathrm{~h} 3 \mathrm{DD}$ & 10'h01E & 10 h3DB & $10 \mathrm{~h} 3 \mathrm{DF}$ & & 10 h3FB & 10 'h3D9 & 10'h03E & 10 h3F7 \\
\hline d7 & 10' $3 \mathrm{D} 1$ & 10 h025 & $10 \mathrm{~h} 032$ & 10 h3F5 & 10 'h3C3 & 10 h037 & $10 \mathrm{~h} 01 \mathrm{~A}$ & $10 \mathrm{~h} 3 \mathrm{D} 7$ & 10 h023 & 10 h026 \\
\hline $\mathrm{d} 8$ & 10 'h3D3 & 10 h033 & 10 h004 & $10 \mathrm{~h} 01 \mathrm{~F}$ & 10'h3EA & $10 \mathrm{~h} 3 \mathrm{DA}$ & 10' h3EE & 10 h3E1 & $10 \mathrm{~h} 3 \mathrm{D} 7$ & $10 \mathrm{~h} 3 \mathrm{CD}$ \\
\hline d9 & $10 \mathrm{~h} 3 \mathrm{DF}$ & 10h01E & 10'h02B & $10 \mathrm{~h} 032$ & 10 'h039 & $10 \mathrm{~h} 3 \mathrm{~EB}$ & $10 \mathrm{ho11}$ & $10 \mathrm{~h} 01 \mathrm{E}$ & 10'h3D5 & 10' h3E4 \\
\hline d10 & 10 h02D & $10 \mathrm{~h} 3 \mathrm{CE}$ & 10 h028 & 10 h3F6 & 10'h3D0 & $10 \mathrm{~h} 3 \mathrm{D} 5$ & 10h01E & 10 h030 & 10'h01D & 10 ' 3 E 5 \\
\hline d11 & 10 'h3DA & $10 \mathrm{~h} 030$ & $10 \mathrm{~h} 3 \mathrm{DA}$ & & $10 \mathrm{~h} 3 \mathrm{D} 6$ & 10' h3EA & 10 h024 & $10 \mathrm{~h} 013$ & 10'h3EA & $10 \mathrm{~h} 3 \mathrm{~B} 7$ \\
\hline $\mathrm{d} 12$ & 10 h014 & 10'h3D1 & 10'h01A & $10 \mathrm{holc}$ & 10 h002 & $10 \mathrm{~h} 02 \mathrm{~F}$ & $10 \mathrm{~h} 023$ & 10'h3EF & 10'h3DC & 10 h036 \\
\hline $\mathrm{d} 13$ & 10 'h3E2 & 10 h029 & 10'h3E3 & 10 'h3D0 & 10'h3FD & 10 h3E3 & $10 \mathrm{~h} 012$ & 10 'h03E & 10'h3E3 & $10 \mathrm{~h} 016$ \\
\hline d14 & 10 ' $3 \mathrm{DE}$ & $10 \mathrm{~h} 010$ & 10 h030 & 10'h3E2 & 10'h3E4 & $10 \mathrm{~h} 01 \mathrm{D}$ & $10 \mathrm{~h} 026$ & 10 h004 & 10'h025 & $10 \mathrm{~h} 027$ \\
\hline d15 & $10 \mathrm{~h} 03 \mathrm{~A}$ & $10 \mathrm{ho2C}$ & 10'万ЗE7 & 10 h01E & 10'万3D8 & $10 \mathrm{ho23}$ & $10 \mathrm{~h} 3 \mathrm{~F} 4$ & $10 \mathrm{~h} 023$ & 10'ћ3ВС & $10 \mathrm{ho2C}$ \\
\hline d16 & 10 'h3EB & 10 h025 & 10'h3D3 & 10 hoof & 10'h3D8 & 10' h3E2 & 10 h3E5 & 10 h3D5 & 10'h3D2 & $10 \mathrm{~h} 3 \mathrm{D} 7$ \\
\hline 01 & $10 \mathrm{~h} 000^{\circ}$ & & & & & & & $10 \mathrm{~h} 015$ & 10 h008 & $10 \mathrm{~h} 014$ \\
\hline $\mathrm{O} 2$ & 10 h000 & & & & & & & $10 \mathrm{~h} 016$ & 10 hoof & $10 \mathrm{~h} 018$ \\
\hline ndex & 16 h0000 & & & & & & & 16 h1000 & & $16 \mathrm{~h} 0010$ \\
\hline
\end{tabular}

Fig. 9. RTL simulation of the function of a modified rejection-based $16 \times 2$-MIN module.

TABLE IV

APPLICATION-SPECIFIC INTEGRATED CIRCUIT (ASIC) RESULTS FOR THE PROPOSED ARCHITECTURE AND OTHER WORKS.

\begin{tabular}{|c|c|c|c|c|c|c|c|c|c|c|}
\hline & This work & {$[21]$} & {$[22]$} & {$[23]$} & {$[24]$} & [25] & {$[26]$} & {$[27]$} & {$[28]$} & {$[5]$} \\
\hline Process technology & $28 \mathrm{~nm}$ & $28 \mathrm{~nm}$ FD-SOI & $28 \mathrm{~nm}$ CMOS & $28 \mathrm{~nm} \mathrm{CMOS}$ & $28 \mathrm{~nm}$ CMOS & $28 \mathrm{~nm} \mathrm{CMOS}$ & $65 \mathrm{~nm} \mathrm{CMOS}$ & $65 \mathrm{~nm} \mathrm{CMOS}$ & $65 \mathrm{~nm} \mathrm{CMOS}$ & $90 \mathrm{~nm} \mathrm{CMOS}$ \\
\hline LDPC code & IEEE 802.11 & $(2048,1723)$ & $(30000,26786)$ & $(672,336)$ & IEEE 802.11ad & IEEE 802.11ad & $(672,546)$ & $(2048,1723)$ & $(2048,1723)$ & IEEE 802.11 \\
\hline Algorithm & Min-sum & Finite-alphabet & Adaptive degeneration & Min-sun & - & $\begin{array}{c}\text { Time-distributed } \\
\text { min-sum }\end{array}$ & Min-sum & Offset min-sum & Split-row & Min-sum \\
\hline$J_{\max }$ & 5 & 5 & 49 & 4 & 15 & 10 & 9 & 8 & 11 & 10 \\
\hline Quanitization bits & $(6,2)$ & 3 & 5 & - & 5 & 5 & 4 & 4 & 5 & 5 \\
\hline$E_{b} / N_{0}[\mathbf{d B}]$ & $\begin{array}{c}2.95-5.5 \\
\text { (@) }=1 \mathrm{e}-7 \text { ) }\end{array}$ & $\begin{array}{c}4.95 \\
\text { (@) } \mathrm{BER}=1 \mathrm{e}-7 \text { ) }\end{array}$ & $\begin{array}{c}10.1 \\
(\text { (@ BER = 1e-15) }\end{array}$ & $\begin{array}{c}4 \\
\text { (@ } @ \text { BER = 1e-6) }\end{array}$ & $\begin{array}{c}4.4-5 \\
(\text { (@ BER = 1e-6) }\end{array}$ & $\begin{array}{c}4.6-5.4 \\
\text { (@ER }=1 \mathrm{e}-6)\end{array}$ & - & $\begin{array}{c}4.25 \\
\text { (@ } \mathrm{BER}=1 \mathrm{e}-7)\end{array}$ & $\begin{array}{c}4.55 \\
(@ B E R=1 \mathrm{e}-7)\end{array}$ & $\begin{array}{c}3-4.5 \\
\text { (@ } \mathrm{BER}=1 \mathrm{e}-7 \text { ) }\end{array}$ \\
\hline Architecture & Partial-parallel & $\begin{array}{c}\text { (@ BER }=1 \text { (1- }-1) \\
\text { Full-parallel }\end{array}$ & (1) BEK = $=1 \mathrm{e}-1 \mathrm{D})$ & $\begin{array}{c}\text { (e) BER = Ie-0) } \\
\text { Partial-parallel }\end{array}$ & $\begin{array}{c}\text { (1) BEK }=1 \mathrm{e}-0) \\
\text { Parallel }\end{array}$ & $\begin{array}{c}(\text { (w) BEK }=1 \mathrm{e}-0) \\
\text { Parallel }\end{array}$ & Full-parallel & Partial-parallel & Full-parallel & Partial-parallel \\
\hline Core area $\left[\mathrm{mm}^{2}\right]$ & $0.038-0.160$ & 16.2 & 3.73 & 0.78 & 0.63 & 1.99 & 12.9 & 5.05 & 4.84 & 5.2 \\
\hline$f_{\max }[\mathbf{M H z}]$ & $2500-3300$ & 862 & 373 & 470 & 260 & 202 & 257 & 700 & 195 & 336 \\
\hline Throughput [Gbps] & $3.68-43.29$ & 588 & 200 & 18.4 & $1.5-12$ & $6.78-16.95$ & 160.8 & 13.3 & 36.3 & $1.7-5.13$ \\
\hline Power $[\mathrm{mW}]$ & $138-771$ & 13350 & 301 & 166 & $6.2-180$ & $104-399$ & 5360 & 2800 & 1359 & 451.3 \\
\hline $\begin{array}{l}\text { Normalized throughput } \\
{\left[\mathrm{Gb} / \mathrm{s} / \mathrm{mm}^{2}\right]}\end{array}$ & $96.84-270.56$ & 36.3 & 53.62 & 23.58 & $2.38-19.05$ & $3.41-8.52$ & 12.47 & 2.63 & 7.5 & $0.33-0.99$ \\
\hline
\end{tabular}

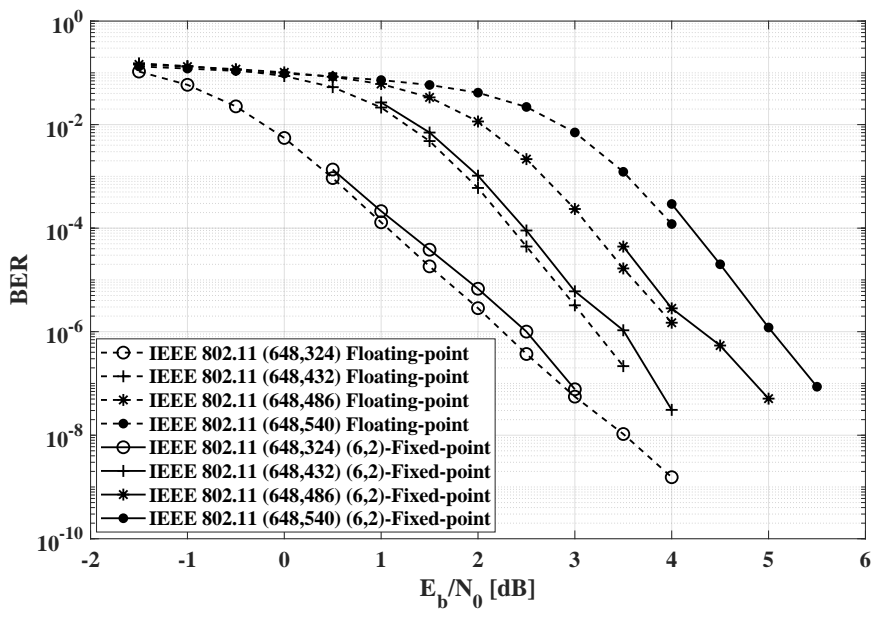

Fig. 10. Effect of quantization on BER performance of IEEE 802.11 codes with LD.

approves the effectiveness of the multi-frame processing in increasing throughput with reasonable hardware overhead.

\section{ACKNOWLEDGMENT}

This work was supported by the German Research Foundation (DFG) project PSSS-FEC, project no. 442607813, application no. LO 2709/1-1, and conducted at IHPmicroelectronics $\mathrm{GmbH}$. The authors are also thankful to

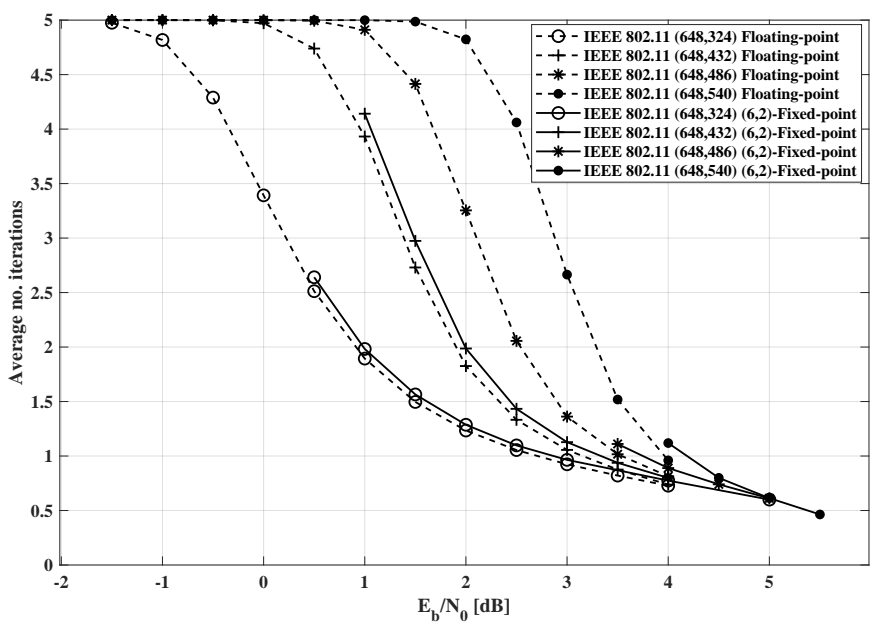

Fig. 11. Average number of iterations for BER performance of Fig. 10.

the support of Brandenburg University of Technology (BTU) Cottbus-Senftenberg, Prof. Dr. Jörg Nolte and Dr. Steffen Büchner.

\section{REFERENCES}

[1] T. Richardson and R. Urbanke, Modern coding theory. Cambridge university press, 2008.

[2] J. Chen and M. P. Fossorier, "Near optimum universal belief propagation based decoding of low-density parity check codes," IEEE Transactions on communications, vol. 50, no. 3, pp. 406-414, 2002. 


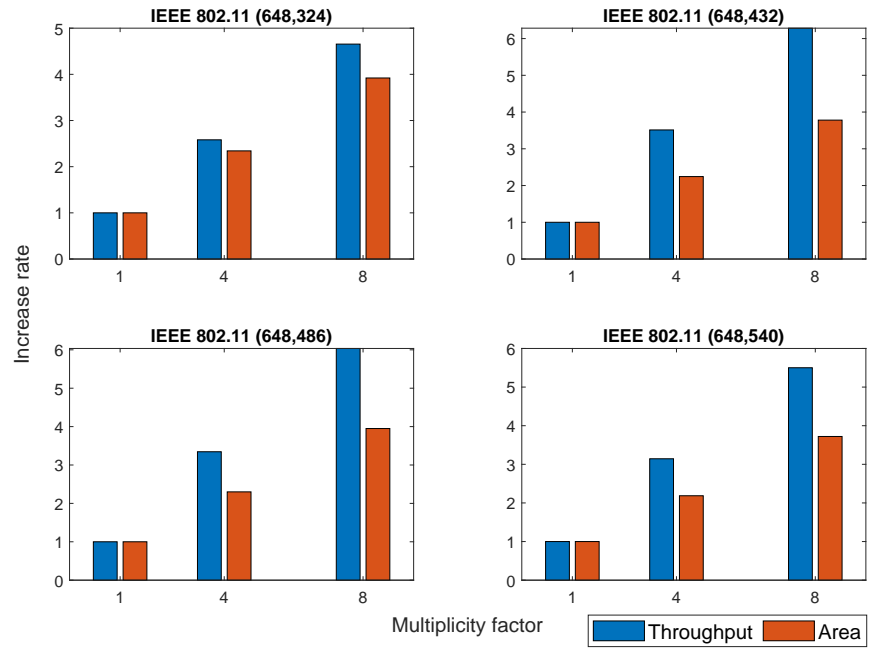

Fig. 12. Increase rate of throughput and chip area with increasing multiplicity factor for four IEEE 802.11 codes.

[3] M. P. Fossorier, M. Mihaljevic, and H. Imai, "Reduced complexity iterative decoding of low-density parity check codes based on belief propagation," IEEE Transactions on communications, vol. 47, no. 5, pp. 673-680, 1999.

[4] A. Hasani, L. Lopacinski, S. Büchner, J. Nolte, and R. Kraemer, "A modified rejection-based architecture to find the first two minima in min-sum-based ldpc decoders," in 2020 IEEE Wireless Communications and Networking Conference (WCNC). IEEE, 2020, pp. 1-6.

[5] S. Kumawat, R. Shrestha, N. Daga, and R. Paily, "High-throughput ldpcdecoder architecture using efficient comparison techniques \& dynamic multi-frame processing schedule," IEEE Transactions on Circuits and Systems I: Regular Papers, vol. 62, no. 5, pp. 1421-1430, 2015.

[6] L. S. Costello and D. J., Error Control Coding, Fundamentals and Applications, 2nd ed., 2004.

[7] M. M. Mansour and N. R. Shanbhag, "Memory-efficient turbo decoder architectures for ldpc codes," in IEEE Workshop on Signal Processing Systems. IEEE, 2002, pp. 159-164.

[8] A. Hasani, L. Lopacinski, S. Büchner, J. Nolte, and R. Kraemer, "A modified shuffling method to split the critical path delay in layered decoding of qc-ldpc codes," in 2019 IEEE 30th Annual International Symposium on Personal, Indoor and Mobile Radio Communications (PIMRC). IEEE, 2019, pp. 1-6.

[9] Chin-Long Wey, Ming-Der Shieh, and Shin-Yo Lin, "Algorithms of Finding the First Two Minimum Values and Their Hardware Implementation," IEEE Trans. Circuits Syst. I Regul. Pap., vol. 55, no. 11, pp. 3430-3437, dec 2008.

[10] X. Chen, Q. Huang, S. Lin, and V. Akella, "FPGA-based low-complexity high-throughput tri-mode decoder for quasi-cyclic LDPC codes," in 2009 47th Annu. Allert. Conf. Commun. Control. Comput. IEEE, sep 2009, pp. 600-606.

[11] L. G. Amaru, M. Martina, and G. Masera, "High Speed Architectures for Finding the First two Maximum/Minimum Values," IEEE Trans. Very Large Scale Integr. Syst., vol. 20, no. 12, pp. 2342-2346, dec 2012.

[12] Qian Xie, Zhixiang Chen, Xiao Peng, and S. Goto, "A sorting-based architecture of finding the first two minimum values for LDPC decoding," in 2011 IEEE 7th Int. Colloq. Signal Process. its Appl. IEEE, mar 2011, pp. 95-98.

[13] W. Cui, Y. Yang, X. Jiang, and S. Kim, "Modified tree structure approach for finding the first two minimum values," in 2014 IEEE China Summit Int. Conf. Signal Inf. Process. IEEE, jul 2014, pp. 563-567.

[14] X.-y. Shih, C.-z. Zhan, and C.-h. Lin, "An $8.29 \mathrm{mmP} 52 \mathrm{~mW}$ MultiMode LDPC Decoder Design for Mobile WiMAX System in 0.13 "m CMOS Process," vol. 43, no. 3, pp. 672-683, 2008.

[15] Y. Lee, B. Kim, J. Jung, and I.-C. Park, "Low-Complexity Tree Architecture for Finding the First Two Minima," IEEE Trans. Circuits Syst. II Express Briefs, vol. 62, no. 1, pp. 61-64, jan 2015.

[16] I. Tsatsaragkos and V. Paliouras, "Approximate Algorithms for Identifying Minima on Min-Sum LDPC Decoders and Their Hardware Implementation," IEEE Trans. Circuits Syst. II Express Briefs, vol. 62, no. 8, pp. 766-770, aug 2015.
[17] G. Tzimpragos, C. Kachris, D. Soudris, and I. Tomkos, "A Low-Latency Algorithm and FPGA Design for the Min-Search of LDPC Decoders," in 2014 IEEE Int. Parallel Distrib. Process. Symp. Work. IEEE, may 2014, pp. 269-274.

[18] J. H. Lee and M. H. Sunwoo, "Low-Complexity First-Two-MinimumValues Generator for Bit-Serial LDPC Decoding," IEEE Trans. Circuits Syst. II Express Briefs, vol. 63, no. 5, pp. 483-487, may 2016.

[19] S. Brown, "Fundamentals of digital logic design with vhdl," 2010.

[20] A. Hasani, L. Lopacinski, and R. Kraemer, "A modified shuffling method to reduce decoding complexity of qc-ldpc codes," 2020.

[21] R. Ghanaatian, A. Balatsoukas-Stimming, T. C. Müller, M. Meidlinger, G. Matz, A. Teman, and A. Burg, "A 588-gb/s ldpc decoder based on finite-alphabet message passing," IEEE Transactions on Very Large Scale Integration (VLSI) Systems, vol. 26, no. 2, pp. 329-340, 2017.

[22] K. Cushon, P. Larsson-Edefors, and P. Andrekson, "Low-power 400gbps soft-decision ldpc fec for optical transport networks," Journal of Lightwave Technology, vol. 34, no. 18, pp. 4304-4311, 2016.

[23] M. Li, J.-W. Weijers, V. Derudder, I. Vos, M. Rykunov, S. Dupont, P. Debacker, A. Dewilde, Y. Huang, L. Van der Perre et al., "An energy efficient $18 \mathrm{gbps}$ ldpc decoding processor for 802.11 ad in $28 \mathrm{~nm}$ cmos," in 2015 IEEE Asian Solid-State Circuits Conference (A-SSCC). IEEE, 2015, pp. 1-5.

[24] M. Weiner, M. Blagojevic, S. Skotnikov, A. Burg, P. Flatresse, and B. Nikolic, "27.7 a scalable 1.5-to-6gb/s 6.2-to-38.1 mw ldpc decoder for $60 \mathrm{ghz}$ wireless networks in $28 \mathrm{~nm}$ utbb fdsoi," in 2014 IEEE International Solid-State Circuits Conference Digest of Technical Papers (ISSCC). IEEE, 2014, pp. 464-465.

[25] M. Milicevic and P. G. Gulak, "A multi-gb/s frame-interleaved ldpc decoder with path-unrolled message passing in 28-nm cmos," IEEE Transactions on Very Large Scale Integration (VLSI) Systems, vol. 26, no. 10, pp. 1908-1921, 2018.

[26] P. Schläfer, N. Wehn, M. Alles, and T. Lehnigk-Emden, "A new dimension of parallelism in ultra high throughput ldpc decoding," in SiPS 2013 Proceedings. IEEE, 2013, pp. 153-158.

[27] Z. Zhang, V. Anantharam, M. J. Wainwright, and B. Nikolic, "An efficient 10gbase-t ethernet ldpc decoder design with low error floors," IEEE Journal of Solid-State Circuits, vol. 45, no. 4, pp. 843-855, 2010.

[28] T. Mohsenin, D. N. Truong, and B. M. Baas, "A low-complexity message-passing algorithm for reduced routing congestion in ldpc decoders," IEEE Transactions on Circuits and Systems I: Regular Papers, vol. 57 , no. 5 , pp. 1048-1061, 2010.

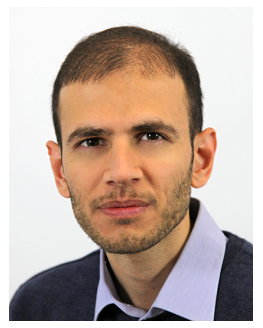

Alireza Hasani received both his B.Sc. and M.Sc. degrees in communication systems from Iran university of science and technology (IUST), Tehran, Iran. He then joined Brandenburg university of technology (BTU), Cottbus, Germany to pursue his study as a Ph.D. student. During that time, he conducted research on channel coding for high-throughput communications systems with collaboration of IHP, Frankfurt (Oder), Germany. His interest is, in general, in any kind of industrial-oriented research in the field of communication systems.

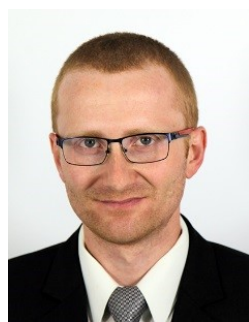

Lukasz Lopacinski received his M.Sc. degree in computer science from West Pomeranian University of Technology, Szczecin, Poland, in 2009 and his Ph.D. degree from Brandenburg University of Technology Cottbus-Senftenberg, Germany, in 2017. From 2007 to 2013, he worked in industrial companies in the field of embedded systems and wireless communication. From 2013 to 2016, he was a research assistant with the Brandenburg university of technology (BTU), Cottbus, Germany. Since 2016, he is working in IHP Microelectronics in Frankfurt 


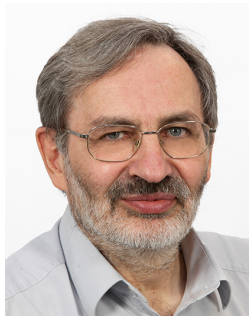

Rolf Kraemer received his diploma and Ph.D. from RWTH Aachen in electrical engineering and computer-science in 1979 and 1985. He joined the Philips research laboratories in 1985 where he worked in different positions and responsibilities. In 1998 he became a professor at the technical university of Cottbus with the joint appointment of the department head of wireless systems at the IHP in Frankfurt (Oder). In the IHP he leads a research department with approximately 70 researchers in topics of high-speed wireless communication, contextaware middleware, sensor networks as well as embedded processors for encryption, and protocol acceleration. 